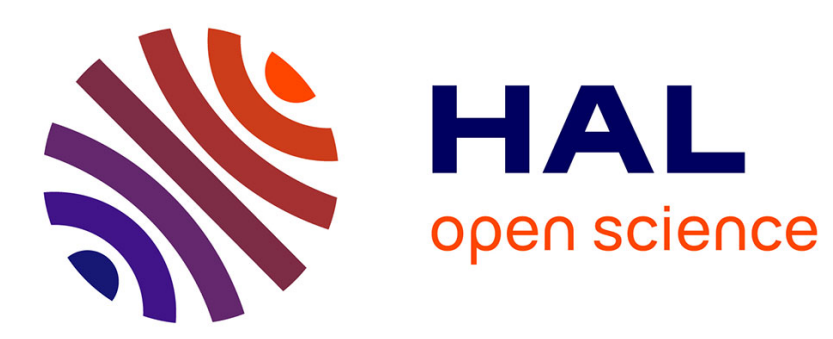

\title{
Imaging of auto-oscillating vocal folds replicas with left-right level difference due to angular asymmetry
}

\author{
Anne Bouvet, Isao Tokuda, Xavier Pelorson, Annemie van Hirtum
}

\section{To cite this version:}

Anne Bouvet, Isao Tokuda, Xavier Pelorson, Annemie van Hirtum. Imaging of auto-oscillating vocal folds replicas with left-right level difference due to angular asymmetry. Biomedical Signal Processing and Control, 2021, 63, pp.102154. 10.1016/j.bspc.2020.102154 . hal-02922361

\section{HAL Id: hal-02922361 \\ https://hal.science/hal-02922361}

Submitted on 26 Aug 2020

HAL is a multi-disciplinary open access archive for the deposit and dissemination of scientific research documents, whether they are published or not. The documents may come from teaching and research institutions in France or abroad, or from public or private research centers.
L'archive ouverte pluridisciplinaire HAL, est destinée au dépôt et à la diffusion de documents scientifiques de niveau recherche, publiés ou non, émanant des établissements d'enseignement et de recherche français ou étrangers, des laboratoires publics ou privés. 


\footnotetext{
${ }^{1}$ Presented in partial form at MAVEBA 2019: 11th International Workshop on Models and Analysis of Vocal Emissions for Biomedical Applications (Florence, Italy, December 17-19, 2019).
} 


\title{
Imaging of auto-oscillating vocal folds replicas with left-right level difference due to angular asymmetry
}

\author{
Anne Bouvet \\ LEGI, UMR CNRS 5519, Grenoble Alpes University, France \\ Isao Tokuda \\ Dep. Mech. Eng., Ritsumeikan Univ., Nojihigashi, Kusatsu, Shiga 525-8577, Japan \\ Xavier Pelorson, Annemie Van Hirtum ${ }^{1}$ \\ LEGI, UMR CNRS 5519, Grenoble Alpes University, France
}

\begin{abstract}
Vertical level difference due to vertical angular asymmetry (up to 20²) between the normal (right) and tilted (left) vocal fold (VF) is experimentally assessed in the framework of unilateral vocal fold paralysis. The upstream pressure and high-speed vibration imaging for different camera view angles are assessed during steady-state auto-oscillation of three different mechanical VF replicas. A global image-based correlation allows to assess the oscillation frequency independently from the camera view angle. Resulting oscillation frequencies matches the ones obtained from aerodynamic data. Mean vibration cycles obtained from videokymographic line-scans at different positions along the posterior-anterior direction are analysed. Left-right VF displacement phase difference, axis shift and left-right mucosal wave phase difference are identified as essential parameters quantifying angular asymmetry. The influence of the degree of angular asymmetry, scan position and replica is discussed.
\end{abstract}

Keywords: Image-based correlation, Vibration asymmetry, Videokymography, Mucosal wave, Unilateral vocal fold paralysis

\footnotetext{
${ }^{1}$ annemie.vanhirtum@univ-grenoble-alpes.fr
} 


\section{Introduction}

Unilateral vocal fold paralysis (UVFP) is a common vocal fold (VF) pathology, reported for instance to affect about $0.5 \%$ of the population in England $[1,2,3]$. In about $83 \%$ of UVFP cases dysphonia is found to reduce the quality and performance of the voice $[1,2,3]$. Clinical UVFP examinations often reveal an air escape due to left-right VF asymmetries with respect to VF's shape, tension and positioning $[1,4,5]$. Despite the continuous advancement of measurement techniques $[6,7,8,9]$ quantitative accurate in-vivo clinical data assessment on human speakers remains tedious, which hampers a systematic assessment of the influence of these asymmetries on voice properties. As a result, a consensus concerning the definition, diagnosis and hence treatment of UVFP remains yet to be achieved [3]. From this perspective, physical studies using deformable vocal folds replicas can provide new insights pertinent to UVFP as they facilitate measurement of variables of interest as well as a systematic variation of the degree of UVFP.

Recently, a physical study using mechanical VF replicas (Fig. 1) was presented in order to experimentally study the effect of air leakage solely due to vertical left-right angular asymmetry on features commonly associated with one) whereas the left VF (the paralyzed one) is tilted with asymmetry angle $\alpha$ in its sagittal plane as illustrated in Fig. 2. Experiments were performed using three different (M5, MRI and EPI) deformable multi-layer silicone VF replicas $[11,12,13,14,15]$ depicted in Fig. 1 [10, 16]. Increasing the asymmetry angle $\alpha$ (up to $25^{\circ}$ ) increases the glottal leakage area (red triangle in Fig. 2). The shape and tension of each VF are unaltered by the imposed asymmetry angle $\alpha$. It followed that the degree of contact between the medial surfaces of the left and right VF decreases and eventually ceases with $\alpha[10,16]$.

30

For all three replicas, it was found $[10,16]$ that when $\alpha$ and hence glottal leakage increases, the oscillation frequency $f_{0}$ reduces monotonically with at least $10 \%$ while both VFs remain in partial contact and even more so when contact between both VFs ceases. This finding offers an interesting new perspective on frequency changes related to UVFP. Indeed, whereas clinical studies generally attribute its symptomatic low frequency voice solely to decreased tension in the paralysed vocal fold $[1,17]$, this physical study [10] 


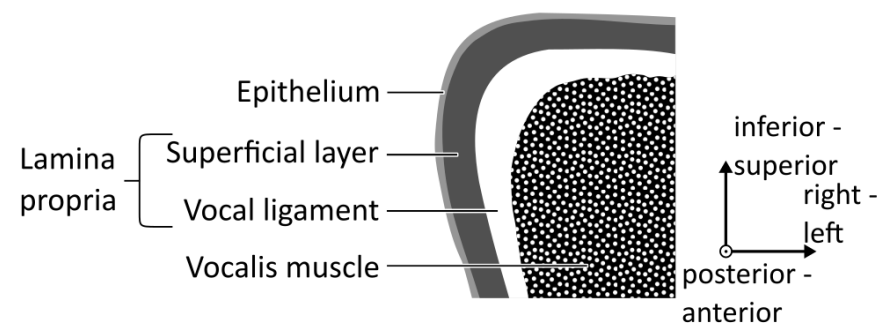

(a) simplified left human VF

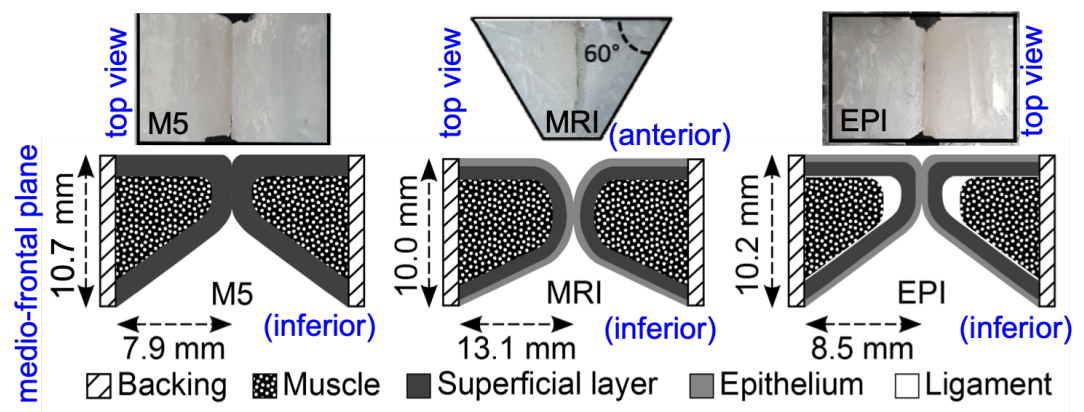

(b) deformable silicone VF replicas

Figure 1: Multi-layered VF structure: a) left human VF in the medio-frontal plane based on [1], b) deformable silicone VF replicas M5, MRI and EPI [10] for $\alpha=0^{\circ}$.

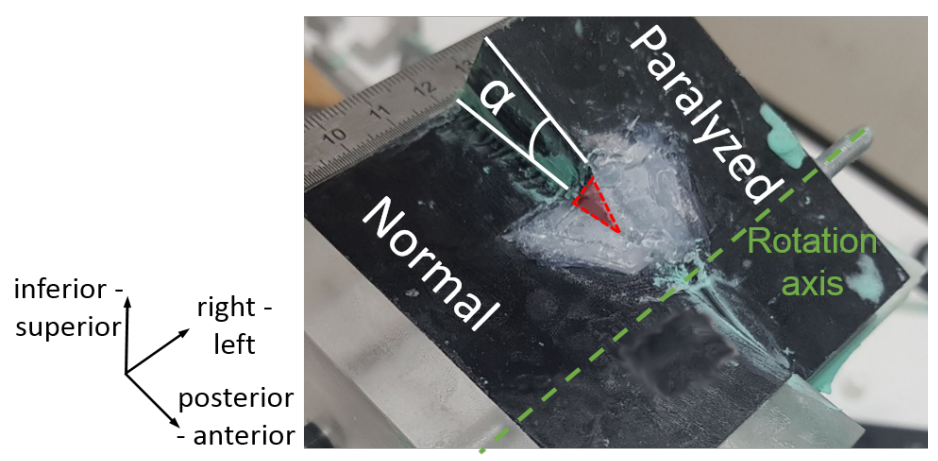

Figure 2: Illustration of imposed asymmetry angle $\alpha$, resulting glottal gap associated with air leakage (red triangle) and fixed rotation axis used for tilting of the left VF in case of the MRI VF replica with $\alpha=20^{\circ}$ [10]. 
shows that frequency reduction might be partly correlated to increased air leakage due to angular asymmetry. In addition, it was found that when full contact is lost so that air leakage occurs, the oscillation quality reduces as the signal-to-noise ratio decreases, the total harmonic distortion rate increases, oscillation threshold pressures at onset and offset increase and higher harmonics become apparent. These observations bear similarities to clinical UVFP voice descriptions of dysphonia (increased harmonic distortion rate cal fatigue and reduced maximum phonation time (increased onset pressure threshold and thus oral airflow during phonation) $[1,18,17]$. As the loss of full glottal contact is seen to trigger these feature changes, experimental observations support voice therapy strategies aiming to remedy glottal closure [18].

Given the described similarities, it is aimed to further investigate the effect of imposing angular asymmetry $\alpha$ on auto-oscillating VF replicas. As in $[10,16]$ different deformable replicas (M5, MRI, EPI) are used in order to assess the generality of observed tendencies. Features discussed in $[10,16]$ were obtained from analysing the subglottal pressure which is an aerodynamic quantity. One might argue that this pressure is difficult to measure directly on human speakers and that aerodynamic observations do not provide a direct measurement of the ongoing vibration. Therefore, the aim of this work is to consider the effect of the imposed angular asymmetry directly on the vibration of auto-oscillating VF replicas using high-speed imaging and to quantify clinically relevant markers. Global and local vibration features are assessed considering instantaneous images (global) or videokymographic (VK) [19] line-scans (local) and the influence of camera view angle (global) and image scan position (local) on quantified features is considered. Local VK features are sought to inform on VF asymmetry.

\section{Glottal replicas}

\subsection{Deformable silicone $V F$ replicas}

70

Oscillation is studied for three different deformable multi-layered molded silicone VF replicas $[10,16]$ - M5, MRI and EPI shown in Fig. 1(b) - The replicas differ in geometry and composition as respectively two (M5), three (MRI) or four (EPI) molding layers are added to a backing layer attaching 
it to a rigid support, i.e. the region outside of the top view frames shown in 75 Fig. 1(b). The M5 VF replica is a two-layer (muscle and superficial layer) reference model following the so called M5 geometrical VF model [20]. The MRI VF replica has a more realistic geometry derived from magnetic resonance imaging data of a human VF $[11,14]$. It has a three-layer structure by adding a third thin and stiff surface layer representing the epithelium to the two-layer structure of the M5 VF replica. The EPI VF replica is obtained by inserting an extremely soft deep layer between the muscle and the superficial layer of the three-layer structure used for the MRI VF replica [13]. The EPI VF replica cast is inspired by the geometrical M5 VF model so that its geometry is a scaled version of the M5 VF replica. A detailed overview of layer thicknesses and Young modulus for each VF replica is provided in [16, 10].

The initial glottal area, i.e. spacing between VFs without airflow, with left-right symmetry $\left(\alpha=0^{\circ}\right)$ yields less than $10 \mathrm{~mm}^{2}$ for each VF replica. Mechanical resonances were identified from frequency response functions. For each replica, a first $f_{1}^{M}$ (quality factor $\approx 11$ ) and second $f_{2}^{M}$ (quality factor $\approx 29$ ) mechanical resonance peak frequency was observed at $142 \pm 5 \mathrm{~Hz}$ and $263 \pm 5 \mathrm{~Hz}$, respectively.

\subsection{Glottal replicas with vertical angular asymmetry angle $\alpha$}

Each deformable silicone VF replica is mounted so that its left VF can be tilted with angle $\alpha$ in the sagittal plane around a fixed rotation axis in the right-left direction. This results in a vertical level difference between the left and right VF parameterised by the imposed angular asymmetry angle $\alpha$ as illustrated in Fig. 2 and in Fig. 3. As the rigid support containing the VF is rotated as well, the elasticity and the shape of each VF is not altered.

As $\alpha$ increases, a triangular glottal air leakage gap (red triangle in Fig. 2) emerges near the posterior VF edge, which is characterised by its area $A$, triangle base $l_{A}$ and triangle height $h_{A}$ indicated in Fig. 3. As $\alpha$ increases the glottal gap extents towards the anterior edge of the replica. It was shown [10] that analytical expressions for $A(\alpha), l_{A}(\alpha)$ and $h_{A}(\alpha)$ can be derived from the other dimensions indicated in Fig. 3, i.e. largest $E$ and smallest $e \mathrm{VF}$ thickness along the inferior-superior direction, length $L$ along the posterioranterior direction, constant distance $a=4.5 \mathrm{~mm}$ between the anterior VF's edge and the rotation axis in the posterior-anterior direction. 


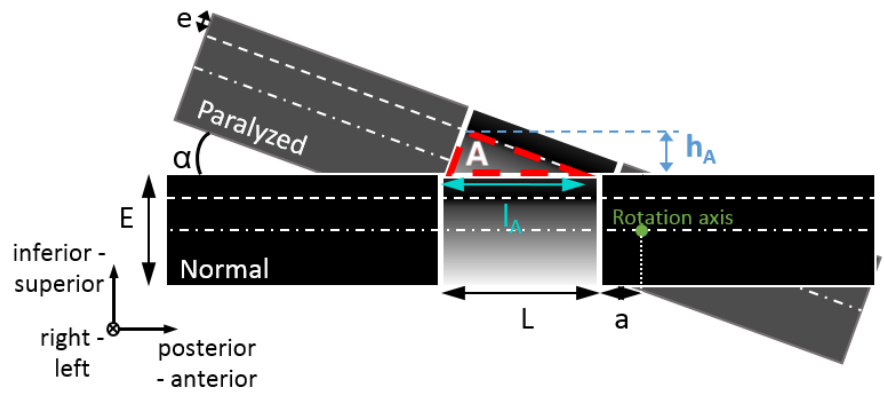

Figure 3: Schematic sagittal side view of glottal replica and dimensions: imposed asymmetry angle $\alpha$, glottal gap (red triangle) characteristics $A, l_{A}, h_{A}$ and VF parameters $E$, $e, L$ and constant rotation axis position $a=4.5 \mathrm{~mm}[10]$.

Three contact regimes are then identified depending on the degree of contact $\left(1-l_{A}(\alpha) / L\right)$ between the medial surfaces of the left and right VF: full contact in regime I $\left(l_{A}=0\right)$, partial contact in regime II $\left(0<l_{A}<L\right)$ and no contact in regime III $\left(l_{A} \geq L\right)$. Two critical angles $\alpha_{I, I I}$ and $\alpha_{I I, I I I}$ are then defined: $\alpha_{I, I I}$ indicating the largest angle for which full contact occurs (shift from regime I to II) and $\alpha_{I I, I I I}$ indicating the largest angle for which partial contact occurs (shift from regime II to III). Critical angles for each of the VF replicas are listed in Table 1. Angles are similar for the M5 and MRI replica and about half these values for the EPI replica.

Table 1: Critical angles $\left(\alpha_{(I, I I)}, \alpha_{(I I, I I I)}\right)$ and contact regime (I, II, III) of $\alpha$.

\begin{tabular}{l||cc||ccc}
\hline Replica & $\alpha_{I, I I}\left[^{\circ}\right]$ & $\alpha_{I I, I I I}\left[^{\circ}\right]$ & I & II & III \\
\hline M5 & 8.1 & 29.2 & $0^{\circ}, 4^{\circ}$ & $10^{\circ}, 16^{\circ}, 20^{\circ}$ & - \\
MRI & 7.8 & 28.8 & $0^{\circ}, 4^{\circ}$ & $10^{\circ}, 20^{\circ}$ & - \\
EPI & 3.0 & 14.0 & $0^{\circ}$ & $4^{\circ}, 10^{\circ}$ & $20^{\circ}$ \\
\hline
\end{tabular}

120

\subsection{Imposed $\alpha$ and relevance to UVFP}

Angular asymmetry angles $\alpha \leq 20^{\circ}$ are experimentally imposed: $\alpha \in$ $\left\{0^{\circ}, 4^{\circ}, 10^{\circ}, 16^{\circ}, 20^{\circ}\right\}$ for the M5 replica and $\alpha \in\left\{0^{\circ}, 4^{\circ}, 10^{\circ}, 20^{\circ}\right\}$ for the MRI and EPI replicas. Following Table 1, contact regime III (no contact) 


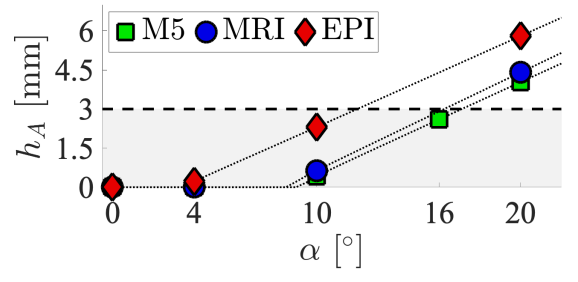

(a) maximum $h_{A}(\alpha)$

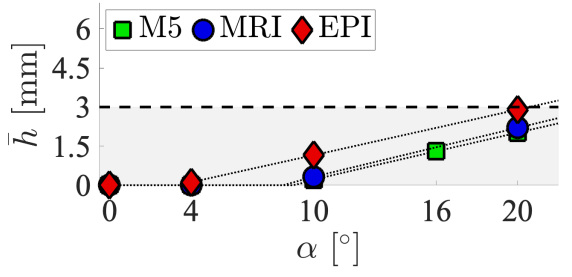

(b) overall $\bar{h}(\alpha)$

Figure 4: Assessed vertical level differences for different replicas M5 ( $\square)$, MRI $(\bigcirc)$ and EPI $(\diamond)$ and range for human UVFP subjects [5] (gray-shaded region up to $3 \mathrm{~mm}$ ): a) maximum $h_{A}(\alpha)$, b) overall $\bar{h}(\alpha)$.

occurs only for the EPI VF replica at $\alpha=20^{\circ}$ as $\alpha_{I I, I I I}>20^{\circ}$ holds for both the MRI and EPI VF replicas. Contact regime I (full contact) and regime II (partial contact) are explored for all three replicas as indicated in Table 1.

In terms of UVFP, the vertical level difference between the superior transverse surfaces of the left and right VF is one of the sought clinical markers, reported to range up to $3 \mathrm{~mm}$ for UVFP subjects [5]. For each replica, two vertical level difference measures are considered, i.e. maximum vertical level difference $h_{A}(\alpha)$ and overall vertical level difference $\bar{h}(\alpha)=A(\alpha) / l_{A}(\alpha)$ so that $\bar{h}(\alpha)=h_{A}(\alpha) / 2$. From Fig. 4 it can be seen that $h_{A}(\alpha)<6 \mathrm{~mm}$ and $\bar{h}(\alpha)<3 \mathrm{~mm}$, respectively twice and once the range (up to $3 \mathrm{~mm}$ ) reported for human subjects. It follows that measurements for $\alpha=20^{\circ}$ fall either just outside $\left(h_{A}\right)$ or just within $(\bar{h})$ the range observed on human subjects. This illustrates the need, reported [3], for a clear definition and precise measurement technology of vertical level difference in order to facilitate the comparison of findings on human subjects.

Regardless of their discrepancy, experimentally assessed maximum $h_{A}(\alpha)$ and overall $\bar{h}(\alpha)$ are both of the order of magnitude observed in human subjects. Consequently, assessed asymmetry angles $\alpha$ allow to investigate for each replica the influence of air leakage due solely to angular asymmetry in a range pertinent to UVFP. Inter-replica variations are then attributed to their different multi-layered structure and shape as outlined in Section 2.1. 


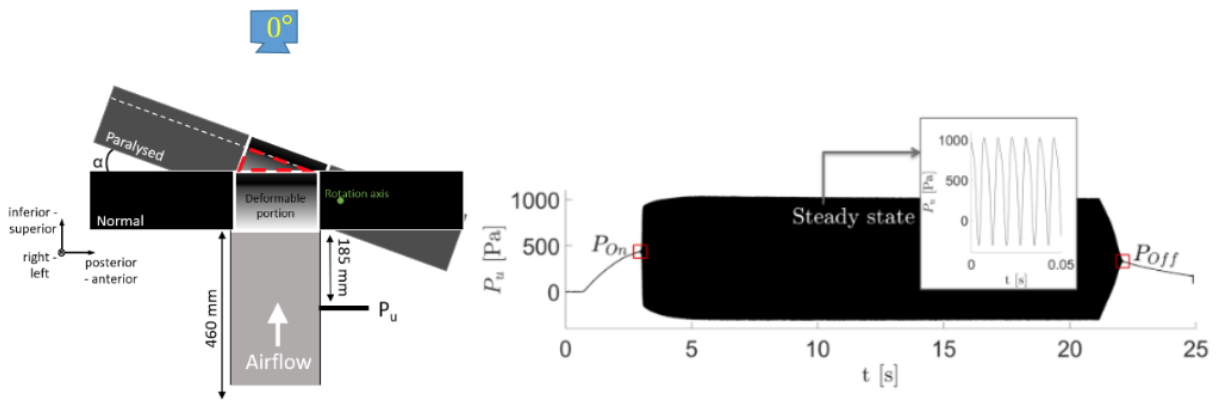

(a) overview of setup for $\theta_{H S}=0^{\circ}$ and $P_{u}(t)$

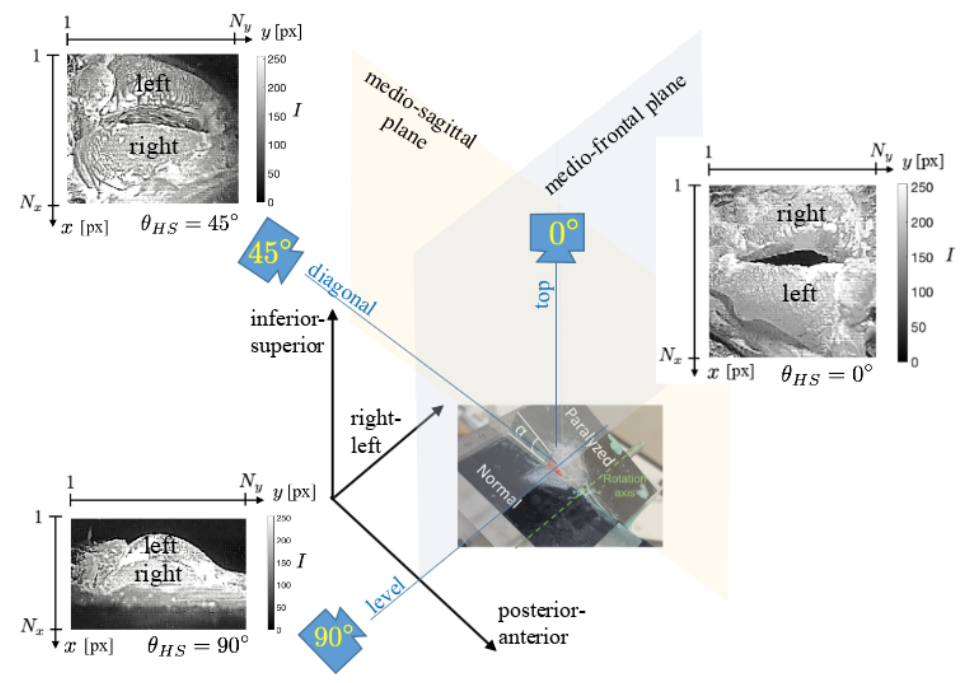

(b) viewing angles $\theta_{H S} \in\left\{0^{\circ}, 45^{\circ}, 90^{\circ}\right\}$

Figure 5: a) Overview of experimental setup and typical experiment: imposed asymmetry angle $\alpha$, upstream pressure $\left(P_{u}\right)$ sensor tap, HS camera (top view $\theta_{H S}=0^{\circ}$ ) and $P_{u}(t)$ indicating auto-oscillation onset, steady state and offset. b) HS camera viewing angles: $\theta_{H S}=0^{\circ}$ (top view), $\theta_{H S}=45^{\circ}$ (diagonal view) and $\theta_{H S}=90^{\circ}$ (level view).

\section{Experimental setup}

Each VF replica with imposed angular asymmetry $\alpha$ is mounted in the experimental setup depicted in Fig. 5 so that a fluid-structure interaction leads to auto-oscillation during which aerodynamic (Fig. 5(a)) and vibration (Fig. 5(b)) data are acquired. A rigid uniform tracheal tube (diameter $16 \mathrm{~mm}$, length $460 \mathrm{~mm}$, acoustic resonance frequency $185 \mathrm{~Hz}$ ) is attached 

Finally, auto-oscillation ceases as $P_{u}$ is reduced below the offset pressure threshold for auto-oscillation $P_{O f f}$. In the following, vibration motion dynamics for each VF replica condition is assessed on $1 \mathrm{~s}$ (corresponding to 4000 subsequent images) of the steady state auto-oscillation indicated in Fig. 5(a).

airtight to the inferior end of the glottal VF replica. Continuous steady airflow (density $\rho_{G}=1.2 \mathrm{~kg} \cdot \mathrm{m}^{-3}$, dynamic viscosity $\mu_{G}=1.8 \times 10^{-5} \mathrm{~Pa} \cdot \mathrm{s}$, temperature $24 \pm 2{ }^{\circ} \mathrm{C}$ ) is provided by an air compressor (Hitachi SC820) connected to a pressure reservoir (volume $0.04 \mathrm{~m}^{3}$ ). The pressure reservoir is filled with acoustic foam in order to avoid parasite acoustic resonances. The compressor is equipped with a pressure regulator (10202U, Fairchild, Winston-salem, NC). A pressure transducer (Kyowa PDS-70GA, accuracy $\pm 5 \mathrm{~Pa}$ ) is positioned in a pressure tap in the tracheal wall, $185 \mathrm{~mm}$ upstream of the glottal VF replica, in order to measure upstream pressure $P_{u}$ as a function of time $t$ with sampling frequency $10 \mathrm{kHz}$. The setup described so far is similar to the one detailed in [10].

In addition to this, a high speed (HS) camera (Keyence VW-9000, frame rate $4 \mathrm{kHz}$, shutter time $4 \mu \mathrm{s}$ and resolution $240 \mathrm{px} \times 320 \mathrm{px})$ is used to acquire instantaneous VF images, i.e. two dimensional grayscale intensity matrices $I(x, y, t)$ (or $I_{x y}(t)$ in short) with $x$ and $y$ indicating the pixel position as shown in Fig. 5(b). Image sequences are cropped from their original size $240 \times 320$ to the region-of-interest containing the vibrating VF's. It follows that in general $1 \leq x \leq N_{x}$ and $1 \leq y \leq N_{y}$ with $N_{x}$ and $N_{y}$ indicating the number of pixels in the $x$ and $y$ dimension, respectively. A single HS camera is rotated in the medio-frontal plane, i.e. around the posterioranterior axis, to observe the VFs (distance $7 \mathrm{~cm}$ ) from three distinct viewing angles $\theta_{H S}$, taken as the angle between the observation direction and the medio-sagittal plane, depicted in Fig. 5(b): top view $\theta_{H S}=0^{\circ}$, horizontal level view $\theta_{H S}=90^{\circ}$ and diagonal view $\theta_{H S}=45^{\circ}$.

The fluid-structure interaction leading to auto-oscillation is illustrated plotting $P_{u}(t)$ in Fig. 5(a). As airflow is supplied, upstream pressure $P_{u}$ gradually increases until auto-oscillation starts as $P_{u}$ reaches the onset pressure threshold for auto-oscillation $P_{O n}$. Steady state auto-oscillation is then observed for several seconds during which mean upstream pressure $\bar{P}_{u} \approx P_{O n}$. Finally, auto-osillation ceases as $P_{u}$ is reduced below the offset pressure (1) 


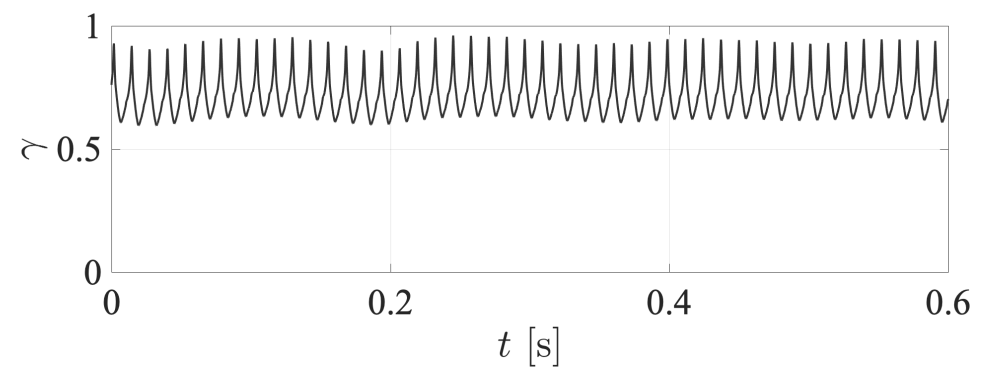

(a) time trace $\gamma(t)$

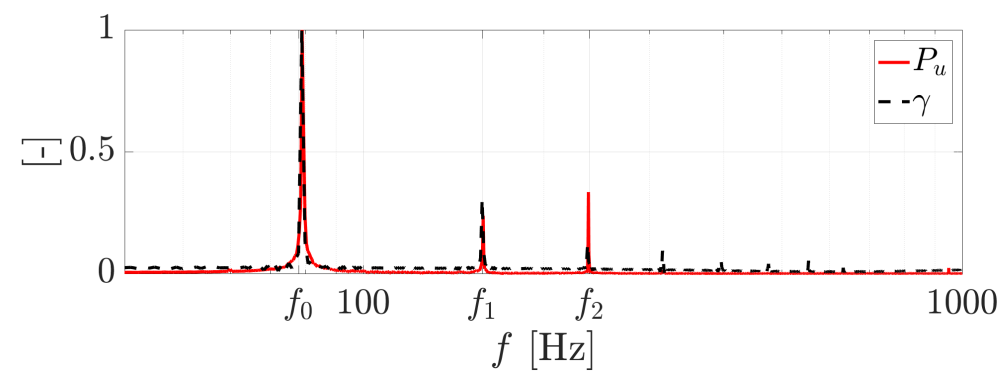

(b) normalised spectra of $\gamma(t)$ and $P_{u}(t)$

Figure 6: Illustration for the EPI VF replica with $\alpha=20^{\circ}$ and $\theta_{H S}=90^{\circ}$ of: a) imagebased correlation $\gamma(t)$, b) Normalised amplitude spectra from $\gamma(t)$ (dashed line) and from upstream pressure $P_{u}(t)$ (full line) and harmonic frequencies $f_{0,1,2}$. The frequency scale is logarithmic.

\section{Image analysis}

\subsection{Global: image correlation}

For each viewing angle $\theta_{H S}$, the image correlation coefficient $\gamma(t)$ between image $I_{x y}(t)$ and reference image $J_{x y}=I_{x y}\left(t_{r e f}\right)$ is calculated. Consequently, an image-based correlation (IC) time series $\gamma(t)$ is obtained quantifying the instantaneous degree $(|\gamma \leq 1|)$ of similarity to $J_{x y}$ as:

$$
\gamma(t)=\frac{\sum_{x} \sum_{y}\left(I_{x y}(t)-\bar{I}\right)\left(J_{x y}-\bar{J}\right)}{\sqrt{\left(\sum_{x} \sum_{y}\left(I_{x y}(t)-\bar{I}\right)^{2}\right)\left(\sum_{x} \sum_{y}\left(J_{x y}-\bar{J}\right)^{2}\right)}}
$$



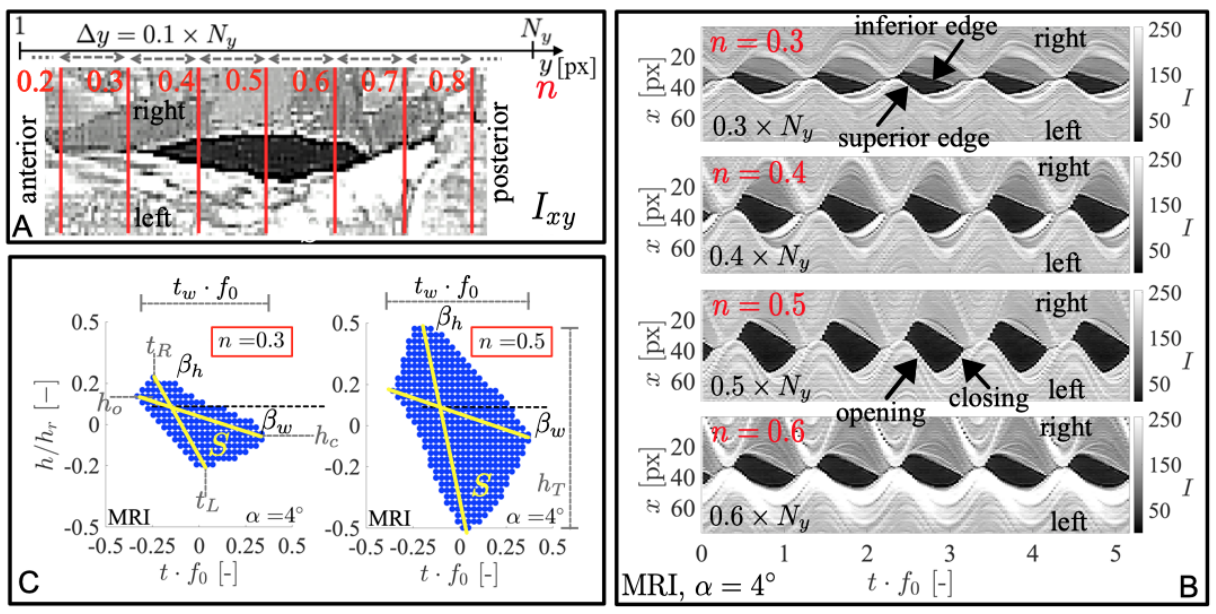

Figure 7: Illustration of VK analysis for top view images $\theta_{H S}=0^{\circ}$ : line extraction of instantaneous image $I_{x y}$ for $y=n \times N_{y}$ (frame A), 5 periods of each multi-VK image set (frame B) and feature extraction on mean aperture (frame $\mathrm{C}$ ).

where averaged image intensities are obtained as $\bar{I}=\sum_{x y} I_{x y} / N$ and $\bar{J}=$ $\sum_{x y} J_{x y} / N$ with $N=N_{x} \times N_{y}$ the number of pixels in each image. A time trace of $\gamma(t)$ is illustrated in Fig. 6(a) (EPI replica, $\alpha=20^{\circ}, \theta_{H S}=90^{\circ}$ ).

Normalised amplitude spectra of $\gamma(t)$ are considered from which oscillation frequency $f_{0}$ is derived. As an example, IC (from $\gamma(t)$ ) and aerodynamic (from $P_{u}(t)$ ) amplitude spectra are plotted in Fig. 6(b) (EPI replica, $\alpha=20^{\circ}$, $\theta_{H S}=90^{\circ}$ ). Harmonic frequencies $f_{1}$ and $f_{2}$ are indicated.

\subsection{Local: left-right videokymographic vibration parameters}

High-speed videokymography (VK) is a common technique to observe the VF's vibration dynamics from top view imaging $\left(\theta_{H S}=0^{\circ}\right)$ on human subjects [19] as well as on VF replicas [14]. Whereas the proposed IC analysis Eq. (1) results in a global image feature $\gamma(t)$, VK allows a local analysis as it scans in each image $I_{x y}(t)$ along the right-left $x$-direction pixels with constant posterior-anterior $y$-position. As tilting $\left(\alpha>0^{\circ}\right)$ of the left VF might provoke an asymmetry in the displacement of each single VF along the $y$-axis, line extraction in $I_{x y}$ is done for several $y$-positions $n$ so that $y=n \times N_{y}$ 
with $n \in\{0.2, \ldots, 0.8\}$ as indicated by the vertical lines (red) in part A of Fig. 7. It follows that the line-scan at $y=0.2 \times N_{y}$ is near the anterior edge and the rotation axis. The line-scan at $y=0.8 \times N_{y}$ is near the posterior edge where the glottal gap emerges for $\alpha>\alpha_{(I, I I)}$ as outlined in Section 2.2. Consecutive line scans in time result in a set of VK images associated with each $y$-position as illustrated in frame B of Fig. 7. Shown VK images allow to visualise the displacement of the left and right VF towards and away from each other during the closing and opening portion of the glottal cycle. It is noted that for $\alpha>\alpha_{I, I I}$ apparent full closure in the VK images might mask air leakage due to the glottal gap in the medio-sagittal plane.

The analysis of each VK image relies on a common threshold-based segmentation of the VF edges and aperture for each cycle. The cycle's mean aperture is determined as the set of pixels surrounding the centroids recurring in at least $80 \%$ of individual cycles. As such each VK image condition (replica, angular asymmetry $\alpha$ and position $n$ ) is characterised by its mean aperture and its edges visualising the closing and opening of the left and right $\mathrm{VF}$ at that position. Resulting mean apertures for $n=0.3$ and $n=0.5$ are illustrated in frame $\mathrm{C}$ of Fig. 7. Time $t$ is normalised by the oscillation period $1 / f_{0}$ so that each normalised cycle has unit period. The displacement from the centroid $h$ is normalised by the maximum aperture amplitude $h_{r}$ for all $n$ so that $-0.5 \leq h / h_{r} \leq 0.5$. These normalisations facilitate comparison between different conditions determined by the used replica, $\alpha$ and position $n$.

Glottal aperture parameters and left-right vibration asymmetry are quantified on the mean aperture and its extrema illustrated in frame C of Fig. 7.

\subsubsection{Glottal aperture measures}

The glottal aperture waveform is characterised by its area $S$. The open quotient is given by the duration of the open portion $t_{w} \cdot f_{0} \leq 1$ as the normalized cycle period has unit length. It follows that the closed portion in the VK image has duration $1-t_{w} \cdot f_{0}$. The skewness of the aperture is expressed considering angles $\beta_{w}$ and $\beta_{h}$ with respect to the direction of the normalised time axis. Angle $\beta_{w}$ is determined by the diagonal direction associated with aperture extrema along the time axis. Hence $\beta_{w} \neq 0^{\circ}$ indicates asymmetry in the displacement of the left and right VF at the beginning $\left(h_{o}\right)$ and end $\left(h_{c}\right)$ of the open portion in each VK image. Angle $\beta_{h}$ is determined by the 
diagonal direction associated with aperture extrema along the displacement axis $h / h_{r}$. Hence $\beta_{h} \neq 90^{\circ}$ indicates asymmetry with respect to the timing at which the displacement of inferior $L$ and $R$ fold edges becomes smaller than the displacement of their respective superior edges.

\subsubsection{Left-right vibration asymmetry parameters}

The angle $\beta_{h}$ is related to the left-right phase asymmetry $P A$ [21], which quantifies the normalized phase delay between the left and right VFs as,

$$
P A=\frac{t_{L}-t_{R}}{t_{w} \cdot f_{0}}
$$

The angle $\beta_{w}$ is related to the axis shift $A S$ quantifying the normalised displacement of the VF during the VK's closed cycle portion [21] as,

$$
A S=\frac{h_{o}-h_{c}}{h_{T}}
$$

with $h_{T}$ the maximal aperture amplitude.

\subsubsection{Left-right mucosal wave asymmetry}

Extreme positions on the mean aperture associated with $\left\{t_{L}, t_{R}, h_{o}, h_{c}\right\}$ in Fig. 7 allow to segment the superior and inferior edges of the right and left VF during the opening (increasing $\left|h / h_{r}\right|$ ) and closing (decreasing $\left|h / h_{r}\right|$ ) portion of the cycle respectively. Noting normalised time $u_{i}=t_{i} \cdot f_{0}$ and normalised edge displacement $s=h / h_{r}$, the wave parameters are determined approximating each edge portion $s\left(u_{i}\right)$ by a Fourier series expansion with $M$ harmonics,

$$
s\left(u_{i}\right)=B_{0}+\sum_{k=1}^{M}\left[A_{k} \sin \left(2 \pi k u_{i}\right)+B_{k} \cos \left(2 \pi k u_{i}\right)\right],
$$

where coefficients $B_{0}, A_{k}$ and $B_{k}$ are estimated using a least square fit [22]. It follows that each mucosal edge wave is described as

$$
s\left(u_{i}\right)=B_{0}+\sum_{k=1}^{M}\left[C_{k} \cos \left(2 \pi k u_{i}+\phi_{k}\right)\right]
$$


with amplitude $C_{k}$ and phase $\phi_{k}$ of the $k^{t h}$ harmonic given as

$$
\begin{aligned}
C_{k} & =\sqrt{A_{k}^{2}+B_{k}^{2}}, \\
\phi_{k} & =-\tan ^{-1}\left(\frac{A_{k}}{B_{k}}\right) .
\end{aligned}
$$

Following [22] the first-order vibration mode $(M=1)$ was used to estimate the wave parameters for each edge. The phases of the inferior and superior edges of the right VF are denoted $\phi_{R, I}$ and $\phi_{R, S}$, respectively. Similarly, phases for the left VF are denoted $\phi_{L, I}$ and $\phi_{L, S}$.

The phase difference between the superior and inferior edge $[23,24]$ for the right and left VF yields

$$
\begin{aligned}
& \phi_{R}=\phi_{R, S}-\phi_{R, I}, \\
& \phi_{L}=\phi_{L, S}-\phi_{L, I} .
\end{aligned}
$$

The phase difference is related to the mucosal wave velocity $c_{R}$ and $c_{L}$ of the right and left $\mathrm{VF}$ as

$$
\phi_{R(, L)}=\frac{2 \pi z_{R(, L)}}{c_{R(L)}},
$$

with $z_{R}$ and $z_{L}$ the distance along the inferior-superior direction between the inferior and superior edges of the right $\left(z_{R}\right)$ and left $\left(z_{L}\right) \mathrm{VF}$. Note that thanks to the applied time normalisation the cycle has unit period so that Eq. 10 does not depend on frequency. Consequently, when $z_{R} \approx z_{L}$, using the assumption that the VF'shape is not affected by $\alpha$, the left-right wave velocity asymmetry is given by the left-right asymmetry in phase difference

$$
\frac{\phi_{R}}{\phi_{L}} \approx \frac{c_{L}}{c_{R}} .
$$

\section{Results}

\subsection{Global analysis}

Oscillation frequencies $f_{0}(\alpha)$ quantified from aerodynamic data $P_{u}(t)$ (empty symbols) and image-based correlation IC $\gamma(t)$ for $\theta_{H S}=90^{\circ}$ (filled symbols) match for all assessed conditions as illustrated in Fig. 8. The maximum discrepancy between different viewing angles $\theta_{H S}$ yields less than $4 \mathrm{~Hz}$, which 


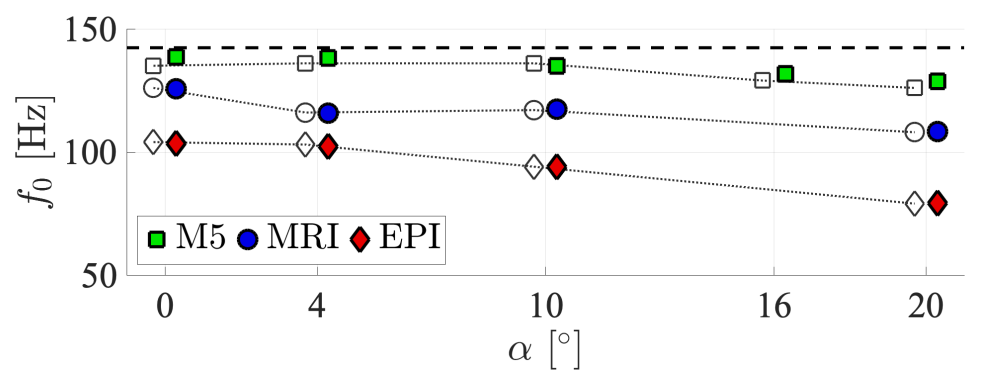

Figure 8: Oscillation frequency $f_{0}(\alpha)$ for VF replicas M5 $(\square)$, MRI $(\bigcirc)$ and EPI $(\diamond)$ from aerodynamic $P_{u}(t)$ (empty symbols) and IC $\gamma(t)$ for $\theta_{H S}=90^{\circ}$ (filled symbols) shifted around $\alpha$ and mechanical resonance frequency $f_{1}^{M}=142 \mathrm{~Hz}$ (horizontal dashed line).

corresponds to the experimental accuracy related to repeatability. Firstly, this confirms that $f_{0}$ can be obtained accurately from vibration imaging using the time series $\gamma(t)$ proposed in Eq. (1). Secondly, it shows that $f_{0}$ quantified from IC $\gamma(t)$ is independent from the camera viewing angle $\theta_{H S}$. It is noted from Eq. (1) that the choice of reference image $J_{x y}$ in the image sequence does not alter the time-dependence of $\gamma(t)$ and hence obtained $f_{0}$.

From Fig. 8 is then seen that both aerodynamic $P_{u}(t)$ and IC $\gamma(t)$ time series reveal the decrease of oscillation frequency $f_{0}$ with increasing $\alpha$ confirming previous observations $[10,14]$. The magnitude of the decrease depends on $\alpha$ as well as on the used VF replica, i.e. $\leq 8 \%$ for $\mathrm{M} 5, \leq 14 \%$ for MRI and $\leq 25 \%$ for EPI, expressing the influence of replica composition and geometry on the vibration.

It was shown on aerodynamic data $P_{u}(t)$ that increasing $\alpha$ is associated with an increased contribution of higher harmonic frequencies to the time trace [10]. Nevertheless, as peak values associated with harmonic frequencies for image-based correlation spectra are found to depend on the imposed camera view angle $\theta_{H S}$ the harmonic content of $\gamma(t)$ is not further quantified. Consequently, also harmonic peak values of aerodynamic and correlation spectra exhibit different harmonic amplitudes, e.g. for $f_{2}$ in Fig. 6(b). 


\begin{tabular}{l||c|c|c}
\hline & mild & moderate & severe \\
\hline M5 & $4^{\circ}$ & $10^{\circ}$ & $16^{\circ}, 20^{\circ}$ \\
MRI & $4^{\circ}$ & $10^{\circ}$ & $20^{\circ}$ \\
EPI & - & $4^{\circ}$ & $10^{\circ}, 20^{\circ}$ \\
\hline
\end{tabular}

Table 2: Angular asymmetry classification for $\alpha>0^{\circ}$ for $\mathrm{VF}$ replicas.

285

290

\subsection{Local analysis}

Vibration observation from VK images is illustrated in Appendix A. The influence of $\alpha$ is easily seen for the MRI replica in Fig. A.16. The impact of the used replica is apparent from Fig. A.17 for both $\alpha=0^{\circ}$ and $\alpha=20^{\circ}$. The impact of scan position $n$ is shown in frame B of Fig. 7 for the MRI VF replica with $\alpha=4^{\circ}$. In the following, measures defined in Section 4.2 are quantified and it is sought to which extent they capture qualitative observations with respect to the used replica (and hence its structure and geometry), scan position $n$ and degree of angular asymmetry $\alpha$. For convenience and considering the vertical level differences plotted in Fig. 4 and critical angles given in Table 1, the angular asymmetry is labelled mild for $0<\alpha \leq \alpha_{I, I I}$, moderate for $\alpha_{I, I I}<\alpha \leq \alpha_{I I, I I I} / 2$ and severe for $\alpha_{I I, I I I} / 2<\alpha$. The resulting angle classification for each replica is summarised in Table 2.

\subsubsection{Glottal aperture measures}

The normalised aperture area $0<S / S_{r} \leq 1$ is plotted in Fig. 9 for each VF replica where the reference area $S_{r}$ is set to its overall maximum. For all replicas, varying $\alpha$ between $0^{\circ}$ and $20^{\circ}$ alters $S / S_{r}$ up to $80 \%$ depending on $n$. Indeed, varying $n$ also induces $S / S_{r}$ to differ up to $80 \%$ depending on the imposed $\alpha$ as in general $S$ increases with $n$ until a maximum is reached after which $S$ reduces. The position of this maximum is more posterior $(n$ increased) for the MRI replica compared to the EPI and M5 replica where the maximum is centered, showing the influence of the replicas geometry. Furthermore, $S$ decreases with $\alpha$ for the M5 replica whereas it increases for MRI and EPI suggesting the influence of the replica composition. The increase with $\alpha$ is gradual for M5. For MRI and EPI the decrease is more pronounced for severe angular asymmetry.

The open quotient $t_{w} \cdot f_{0}<1$ is plotted in Fig. 10. Tendencies agree mostly with those reported for area $S / S_{r}$ indicating that overall larger aper- 


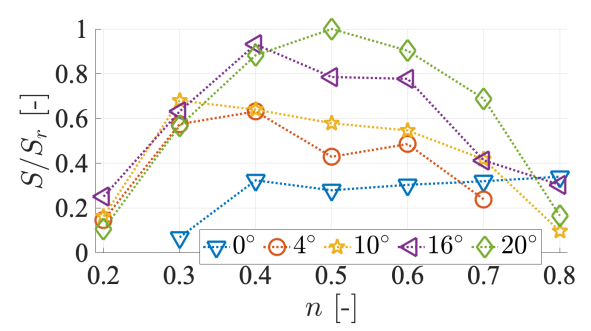

(a) M5

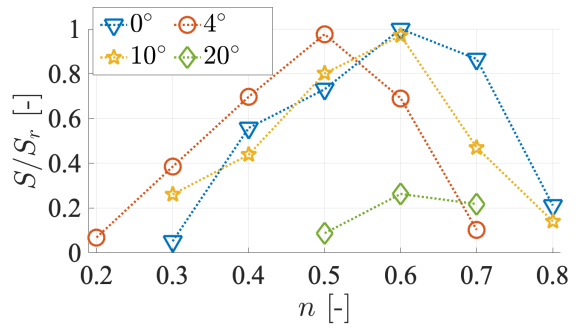

(b) MRI

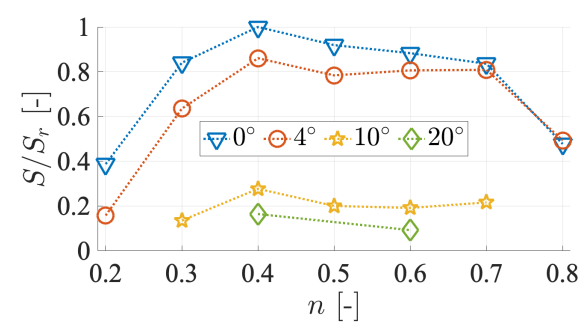

(c) EPI

Figure 9: Normalised aperture area $S(\alpha) / S_{r}$ at different positions $n$ for replicas: a) M5, b) MRI and c) EPI. sensitive to the degree of angular asymmetry $\alpha$ and the replica's geometry. It is noted for MRI and EPI that the reduction of open quotient $t_{w} \cdot f_{0}$ with $\alpha$ is naturally associated with a reduction of the useful scan region along the posterior-anterior direction.

Aperture skewness angles $\beta_{h}$ and $\beta_{w}$ are plotted in Fig. 11 and Fig. 12, respectively. Without angular asymmetry $\left(\alpha=0^{\circ}\right), \beta_{h}$ and $\beta_{w}$ approximate $\beta_{h} \approx 90^{\circ}$ and $\beta_{w} \approx 0^{\circ}$ at center positions $0.3<n<0.7$ expressing symmetrical left-right VF displacement. Imposing angular asymmetry $\left(\alpha>0^{\circ}\right)$ all replicas. Note that $S / S_{r}$ and $t_{w} \cdot f_{0}$ exhibit opposite tendencies with $\alpha$, 


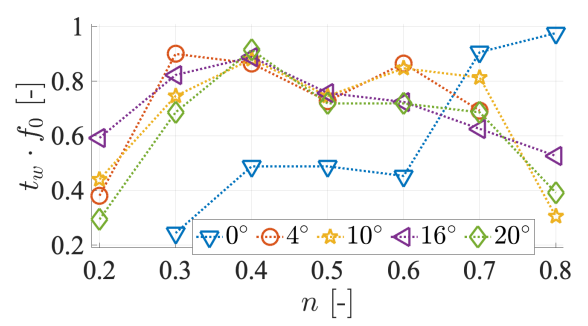

(a) M5

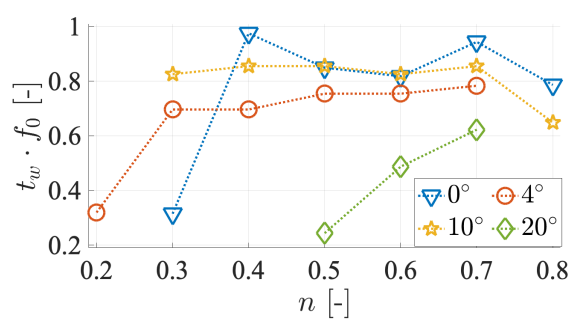

(b) MRI

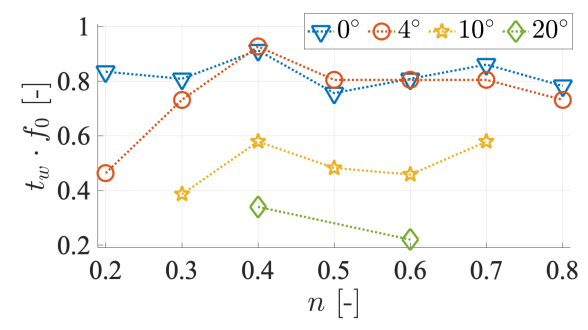

(c) EPI

Figure 10: Open quotient $t_{w} \cdot f_{0}$ as a function of $\alpha$ at different positions $n$ for replicas: a) M5, b) MRI and c) EPI.

i.e. increase for M5 and decrease for MRI and EPI. It follows that both $\beta_{h}$ and $\beta_{w}$ reveal angular asymmetry for $\alpha>0$ indicating that left-right $\mathrm{VF}$ displacement asymmetry and subsequent skewness are inherent features of angular asymmetry. In general, skewness angles $\beta_{h}$ and $\beta_{w}$ do not reflect the severeness of angular asymmetry or scan position $n$ as no general monotonic tendencies with $\alpha$ are distinguished. Nevertheless for $\beta_{w}$ it is noted that such a tendency is observed for the M5 replica as $\beta_{w}$ gradually decreases with both $\alpha$ and $n$, so that $\beta_{w}$ is more prominent for positions near the posterior side which is opposite from the rotation axis. For the EPI replica (and for MRI at large $n$ ) the monotone decrease with $\alpha$ ceases when $\alpha=20^{\circ}$. This is probably due to the small aperture area (Fig. 9) and associated open quotient (Fig. 10) at $\alpha=20^{\circ}$, limiting the decrease for $\beta_{h}$ and $\beta_{w}$.

\subsubsection{Left-right vibration asymmetry}

Following their definitions, left-right phase asymmetry $P A$ (Eq. (2)) is related to $\beta_{h}$ and axis shift $A S$ (Eq. (3)) is related to $\beta_{w}$. Phase asymmetry 


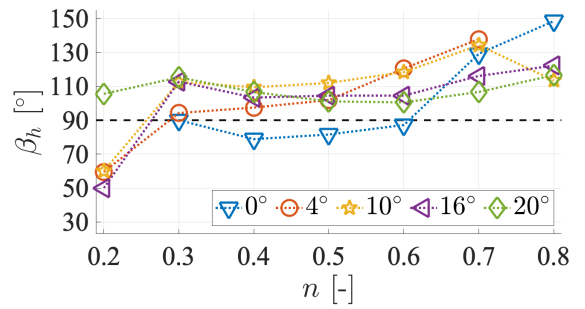

(a) M5

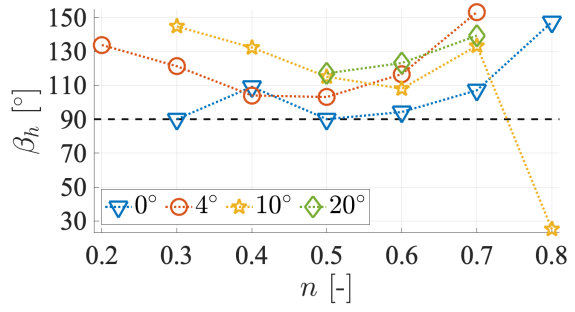

(b) MRI

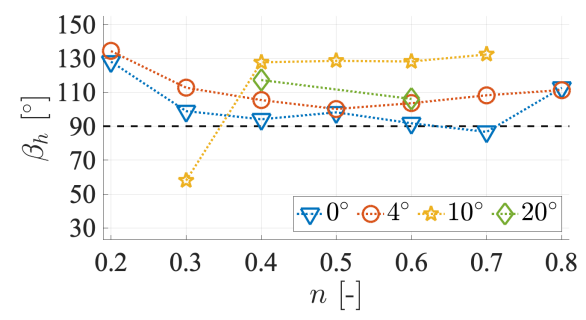

(c) EPI

Figure 11: Aperture angle $\beta_{h}(\alpha)$ at different positions $n$ for replicas: a) M5, b) MRI and c) EPI. The symmetry condition $\beta_{h}=90^{\circ}$ is indicated (dashed line).

$P A$ increases from its symmetry value $P A=0$ with $\alpha$. Observed tendencies for $P A$ are similar to the ones described for $\beta_{h}$ so that $P A$ curves are not plotted. It follows that left-right phase asymmetry can be quantified by either $P A$ or $\beta_{h}$. As $P A>0$ (or equivalently $\beta_{h}>90^{\circ}$ ) holds, this implies that the right VF reaches its maximum displacement followed by the tilted left VF. This agrees with the observation during auto-oscillation experiments that the movement of the right VF entrains the movement of the tilted left VF.

From Fig. 13 it can be seen that for $\alpha>0$ axis shift $A S$ increases from its symmetry value $A S=0$ approximated for $\alpha=0^{\circ}$ for all replicas. For the EPI and MRI replicas findings for $\beta_{w}$ also apply to $A S$ so that $A S$ is related to the severeness of angular asymmetry up to $\alpha=20^{\circ}$. For the M5 replica the dependence on $n$ observed for $\beta_{w}$ is also retrieved for $A S$. Nevertheless, $A S$ does no longer relate to the severeness of angular asymmetry as values for moderate and severe asymmetry overlap. For $\alpha>0^{\circ}$, axis shift $A S>0$ (or $\beta_{w}<0^{\circ}$ ) indicates left-right asymmetrical mucosal wave displacement 


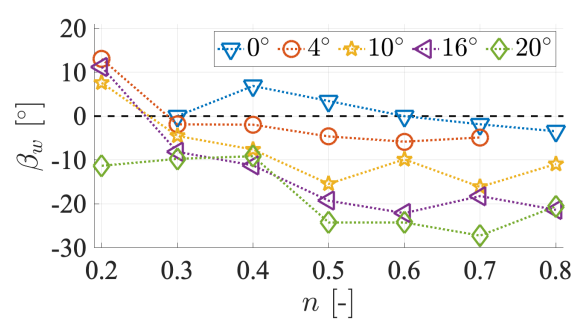

(a) M5

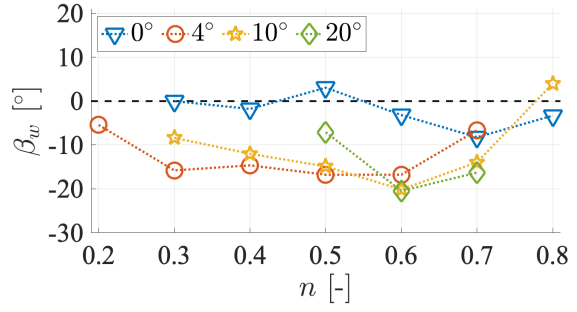

(b) MRI

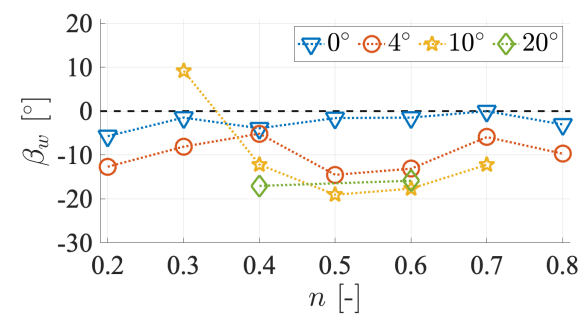

(c) EPI

Figure 12: Aperture angle $\beta_{w}(\alpha)$ at different positions $n$ for replicas: a) M5, b) MRI and c) EPI. The symmetry condition $\beta_{w}=0^{\circ}$ is indicated (dashed line).

during the closed portion. The mucosal wave asymmetry is further assessed in the next section.

\subsubsection{Left-right mucosal wave asymmetry}

The mucosal wave phase difference between the superior and inferior edges of the left $\mathrm{VF} \phi_{L}$ (Eq. 9) normalised by $\phi_{L, r}$, taken as the mean phase difference for all $n$ at symmetry $\left(\alpha=0^{\circ}\right)$, is illustrated in Fig. 14 for the M5 and EPI replicas. It is found that for the MRI and EPI replicas $\phi_{L}$ increases with $\alpha$. The increase is most prominent for moderate and severe asymmetry angles. For the M5 replica $\phi_{L}$ decreases when $\alpha>0^{\circ}$ and values for $\alpha>0^{\circ}$ overlap. The phase difference of the right $\mathrm{VF} \phi_{R}$ is not shown as tendencies are similar as those observed for $\phi_{L}$.

The phase difference ratio between the right and left $\mathrm{VF} \phi_{R} / \phi_{L}$ is plotted in Fig. 15. The ratio also informs on the left-right wave velocity asymmetry $c_{L} / c_{R}$ using Eq. (10). In general, the symmetry condition $\phi_{R} / \phi_{L}=1$ holds 


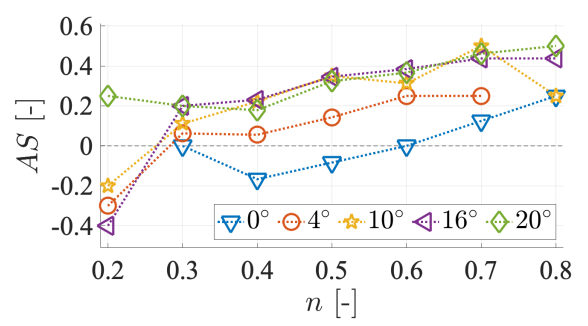

(a) M5

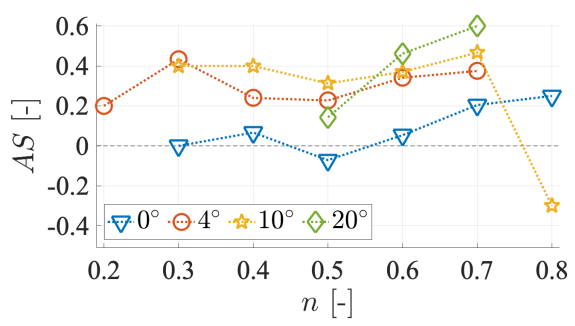

(b) MRI

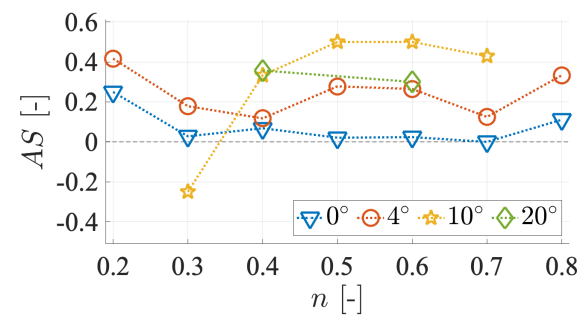

(c) EPI

Figure 13: Axis shift $A S$ as a function of $\alpha$ at different positions $n$ for replicas: a) M5, b) MRI and c) EPI. The symmetry condition $A S=0$ is indicated (dashed line).

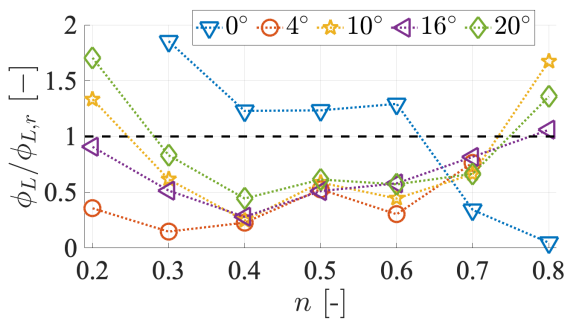

(a) M5

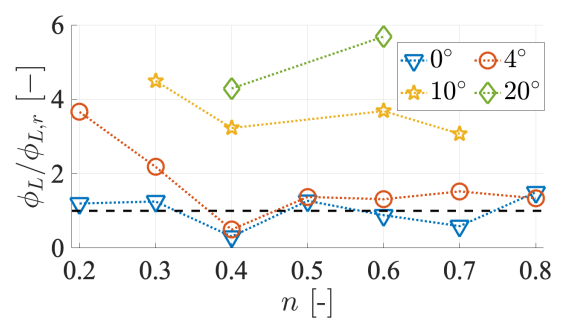

(b) EPI

Figure 14: Normalised phase difference of the left VF $\left(\phi_{L} / \phi_{L, r}\right)$ as a function of $\alpha$ and position $n$ for replicas: a) M5, b) EPI. Ratio 1 is shown (dashed line).

for $\alpha=0^{\circ}$ as phase differences and wave velocities are equal for the left and right $\mathrm{VF}$ as expected. For the MRI and EPI replicas the ratio decreases (up to $60 \%$ ) for mild and moderate asymmetry angles. For severe asymmetry, the ratio increases again towards (MRI) or slightly $(<10 \%)$ above $(\mathrm{EPI})$ unity. For the M5 replica a decrease of $\phi_{R} / \phi_{L}$ with $\alpha$ occurs for moderate angular 


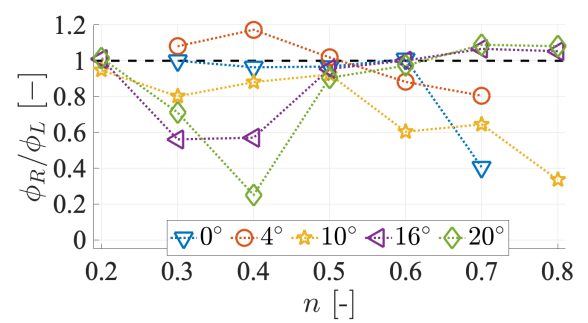

(a) M5

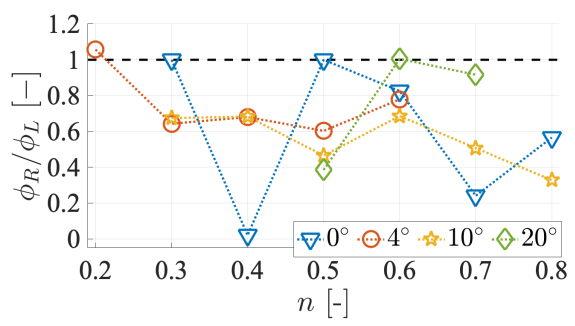

(b) MRI

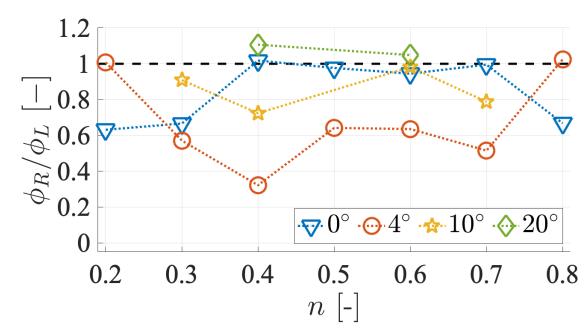

(c) EPI

Figure 15: Left-right phase difference asymmetry $\phi_{R} / \phi_{L}$ as a function of $\alpha$ and position $n$ for replicas: a) M5, b) MRI and c) EPI. Phase symmetry $\phi_{R} / \phi_{L}=1$ is indicated (dashed line).

asymmetries. For severe asymmetries, $\phi_{R} / \phi_{L}$ decreases (up to 80\%) for locations near the anterior axis, whereas they remain around unity for locations near the posterior axis. For mild asymmetries, the ratio $\phi_{R} / \phi_{L}$ also remains near unity. This implies that in general (when $\phi_{R} / \phi_{L}<1$ holds) the phase difference is greater for the tilted left $\mathrm{VF}$ than for the right $\mathrm{VF}$ as $\phi_{L} \geq \phi_{R}$ and therefore $c_{R} \geq c_{L}$ so that the wave velocity associated with the tilted left $\mathrm{VF}$ is lower than the one in the right VF. This is consistent with the observations for $P A$ (and therefore $\beta_{h}$ ), suggesting that the movement of the right VF entrains the left VF; and consistent also with the observation of axis shift $A S$ (and therefore $\beta_{w}$ ) during the closed portion of the cycle. For the M5 replica $\phi_{R} / \phi_{L} \approx 1$ at scan position $n=0.5$ for all $\alpha$ and for the MRI replica the overall discrepancy from unity is smallest at $n=0.6$. It is suggested that these positions, where the discrepancy in left-right phase difference asymmetry with respect to $\alpha$ is smallest, indicate the entrainment position associated with synchronisation and hence the minimum in left-right phase difference asymmetry. The entrainment position is thus centered $(n=0.5)$ for the M5 
replica and is shifted in the posterior direction $(n=0.6)$ due to the geometry of the MRI replica. Note that these positions correspond to maxima in the aperture area $S / S_{r}$. As the area variation of the EPI replica exhibits a broad maximum also the left-right phase difference asymmetry exhibits broader minimum discrepancy for $0.4<n<0.7$ corresponding to the entrainment region. As $\phi_{R} / \phi_{L}$ depends on scan position $n$, a wave-like displacement along the posterior-anterior direction can be observed.

\section{Discussion}

The influence of air leakage due to vertical level difference created by imposing angular asymmetry between the right and tilted left VF is experimentally assessed using high-speed imaging during steady-state auto-oscillation of three deformable silicone VF replicas of different multi-layer composition and geometry. Observations for the MRI and EPI replicas are most relevant to human VF's and hence clinical applications as their structure and shape are based on human VF's. Nevertheless, it is of interest to study and compare vibration observations for all three replicas (M5, MRI and EPI) to understand their differences and similarities.

Image sequences are analysed globally introducing an image-based correlation IC time series $\gamma(t)$ from which the oscillation frequency is obtained regardless of degree of asymmetry, used replica or camera view angle. The shown robustness suggests that $\gamma(t)$ can be applied to clinical imaging data in order to obtain the oscillation frequency. Obtained oscillation frequencies match with frequencies retrieved on the upstream driving pressure $P_{u}(t)$, confirming the use of aerodynamic data to study vibration during autooscillation, and confirming the overall decrease of $f_{0}(\alpha)$ reported in [10]. This is in particular of interest for physical studies during which $P_{u}(t)$ ac430 quisition is often more beneficial than imaging as it is readily accessible and does not demand a direct view of the vibrating VF's, reduces the amount of data storage and hence the amount of data processing and does not involve the use of (expensive) high-speed camera equipment.

Videokymographic images are analysed for different local scan positions $n$ considering mean cycle apertures. To facilitate comparison between different conditions, the oscillation frequencies retrieved from the global image 
analysis are used for time normalisation and the displacement is normalised by the maximum cycle displacement. The influence of angular asymmetry, scan position and replica on left-right VF displacement asymmetry and mucosal wave asymmetry is quantified from common features relevant for clinical studies and for further studies on replicas.

Aperture's area $S / S_{r}$, open quotient $t_{w} \cdot f_{0}$ and superior versus inferior VF edge phase differences $\phi_{L}$ and $\phi_{R}$ reflects the replica's multi-layer composition as these quantities have opposite tendencies for the M5 replica on one hand and the softer MRI and EPI replicas on the other hand. This observation might be related to the minimum in the oscillation onset pressure threshold at $\alpha \approx 10^{\circ}$ found only for the M5 replica [10] indicating that for the M5 replica imposing angular asymmetry at first eases auto-oscillation. For the MRI and EPI replicas, these features can inform on angular asymmetry for severe asymmetries only as for values characterising no, mild and moderate asymmetry overlap. For clinical applications (and when a severe angular asymmetry is suspected) in particular the decrease of $t_{w} \cdot f_{0}$ is a potential indicator of severe angular asymmetry as the open quotient does not depend on scan position $n$ and is known to vary between 0 and 1 . Area $S / S_{r}$ on the other hand is sensitive to scan position $n$ and requires prior knowledge of a reference value $S_{r}$ associated with symmetry $\left(\alpha=0^{\circ}\right)$ which might not be available for an individual human subject. It is noted that for the M5 replica $t_{w} \cdot f_{0}$ only indicates the presence of angular asymmetry $\alpha>0^{\circ}$ and not its severity. Since mild angular asymmetry might also occur for healthy VF's it seems not a pertinent parameter for the clinical study of angular asymmetry. In this case a combination with $S / S_{r}$ is of interest in case reference value $S_{r}$ is known since $S / S_{r}$ relates to the degree of angular asymmetry for the M5 replica.

465 Aperture skewness angle $\beta_{h}>90^{\circ}$ and phase difference $P A>0$ both measure the increase of the phase asymmetry between the right and left $\mathrm{VF}$ with $\alpha$. Aperture skewness angle $\beta_{w}<0^{\circ}$ and axis shift $A S>0$ indicate the increase of left-right asymmetrical mucosal wave displacement with $\alpha$ resulting in a decrease of the left-right phase difference asymmetry $\phi_{R} / \phi_{L}<1$ with

${ }_{470} \alpha$. The tendencies observed for these asymmetry measures hold for all VF replicas so that they are identified as essential angular asymmetry parameters in contrast to previous discussed parameters (area $S / S_{r}$, open quotient $t_{w} \cdot f_{0}$ and phase differences $\phi_{L}$ and $\left.\phi_{R}\right)$ for which tendencies also depend on the replica. Increasing phase difference $\left(P A\right.$ or $\left.\beta_{h}\right)$ indicates that angular asymmetry induces increased entrainment of the tilted left VF by the right 
VF. Decreasing $\beta_{w}$ (or similarly increasing $A S$ ) indicates that angular asymmetry causes an increasingly asymmetric mucosal wave displacement during the closed portion between the left and right VF. Decreasing left-right phase difference asymmetry $\phi_{R} / \phi_{L}$ indicates that angular asymmetry causes the mucosal wave velocity associated with the tilted left VF to lower with respect to the wave velocity of the right $\mathrm{VF}$. It is noted that $\phi_{R} / \phi_{L} \approx 1$ or at least the decrease of $\phi_{R} / \phi_{L} \approx 1$ with $\alpha$ is limited for scan positions $n$ within the entrainment region situated for all replicas near the center position $n=0.5$. It is noted that for the MRI replica with a more realistic shape entrainment is shifted in the posterior direction.

For the MRI and EPI replica phase these asymmetry measures vary gradually with angular asymmetry up to $\alpha<20^{\circ}$ for which their value becomes limited due to the prominent area reduction. Therefore, for clinical applications it seems of interest to consider this parameter set $(P A$ or equivalently $\beta_{h}, A S, \beta_{w}$ and $\left.\phi_{R} / \phi_{L}\right)$ in combination with the open quotient $t_{w} \cdot f_{0}$ in order to assess the degree of angular asymmetry.

For future research on mechanical replicas it is of interest to verify that these findings hold for $\alpha<0^{\circ}$ instead of $\alpha>0^{\circ}$ as considered in the current study. Furthermore, described entrainment and vibration asymmetries are of interest for physical modeling of UVFP. Future clinical studies of UVFP are needed in order to consider a multi-variable analysis of the proposed asymmetry parameter set in order to assess that it allows firstly to detect the presence of UVFP and secondly allows to quantify the degree of UVFP. Note that in case this is the case purely geometrical glottal changes, i.e. tilting, can at least partly explain UVFP symptoms.

\section{Conclusion}

An image-based analysis of steady-state auto-oscillation is presented using VF replicas with imposed angular asymmetry creating vertical level difference as observed in the case of UVFP. A global image-based correlation time series is shown to enable straightforward and robust estimation of the oscillation frequency for all assessed conditions, i.e used replica, angular asymmetry and camera view angle. Videokymographic line scans are then analysed for several scan positions. Angular asymmetry is shown to induce left-right vibration phase asymmetry characterising the entrainment of the left VF by the right VF and a reduced mucosal wave velocity of the left VF 
compared to the its veloctiy associated with the right VF. The extent of this vibration asymmetry induced by angular asymmetry is proposed to be quantified by a set of asymmetry parameters in combination with the open quotient. The use of different replicas has proven to be extremely valuable to identify these general mechanisms and associated parameter sets as general glottal aperture measures such as area and open quotient largely depend on the multi-layer composition of the used replica.

\section{Acknowledgements}

This work was partly supported by ArtSpeech project (ANR-15-CE230024), JSPS International Research Fellowship (SP18205) and JSPS Grantin-Aid for Scientific Research (No. 19H01002). Authors thank Dr. K. Ishimura (Ritsumeikan Univ., Japan) for experimental support.

\section{Appendix A. Illustration of VK images}

Examples of VK images illustrating the influence of imposed asymmetry angle $\alpha$ and the assessed replica, i.e. its shape and composition, on the vibration are shown in Fig. A.16 and Fig. A.17, respectively.

\section{References}

[1] C. Rosen, C. Simpson, Operative techniques in laryngology, SpringerVerlag, 2008.

[2] S. Nouraei, S. Middleton, C. Butler, G. Sandhu, An estimation of the population incidence of adult unilateral vocal fold mobility impairment in England, Logoped Phoniatr Vocol 40 (2015) 93-94.

[3] C. Walton, P. Carding, K. Flanagan, Perspectives on voice treatment for unilateral vocal fold paralysis, Curr Opin Otolaryngol Head Neck Surg 3 (2018) 157-161.

[4] K. Hong, K. Jung, Arytenoid appearance and vertical level difference between the paralyzed and innervated vocal cords, The Laryngoscope 111 (2001) 227-232. 


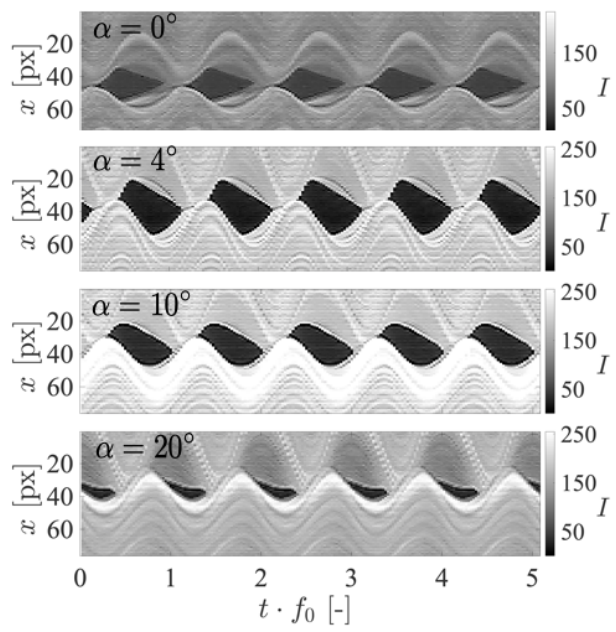

Figure A.16: VK images for the MRI replica along line $y=0.5 \times \Delta y$ (center $0.5 \times N_{y}$ or $n=0.5$ ) for increasing $\alpha=\left\{0^{\circ}, 4^{\circ}, 10^{\circ}, 20^{\circ}\right\}$ (top to bottom).

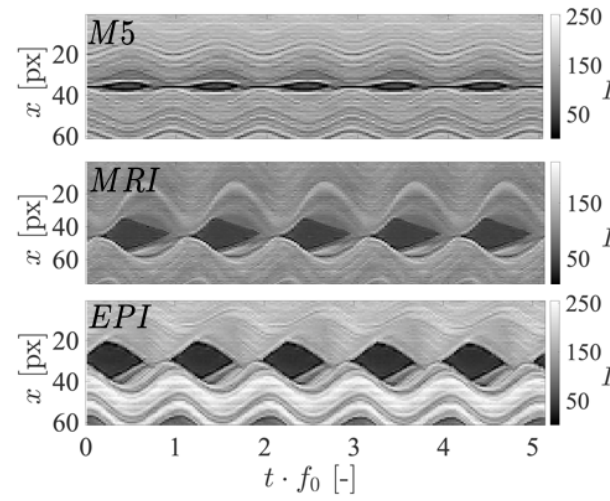

(a) $\alpha=0^{\circ}$
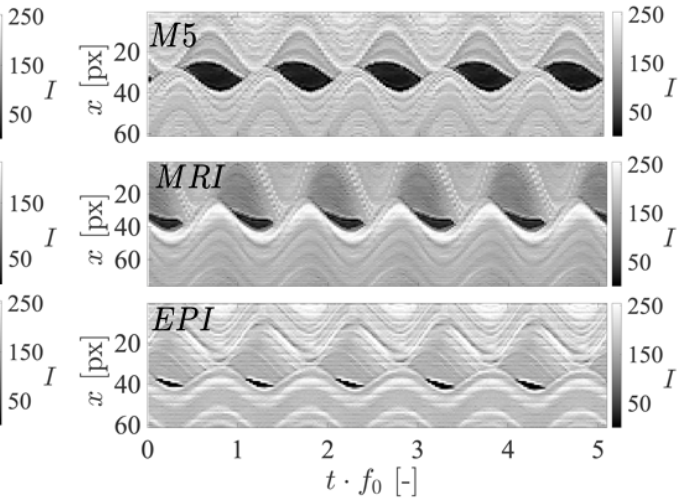

(b) $\alpha=20^{\circ}$

Figure A.17: VK images for the M5, MRI and EPI replica (top to bottom) along line $y=0.5 \times \Delta y\left(\right.$ center $0.5 \times N_{y}$ or $\left.n=0.5\right)$ for: a) $\alpha=0^{\circ}$ and b) $\alpha=20^{\circ}$.

[5] Y. Oyamada, E. Yumoto, K. Nakano, H. Goto, Asymmetry of the vocal folds in patients with vocal fold immobility, Arch Otolaryngol head neck surg 131 (2004) 399-406.

[6] D. Sommer, I. Tokuda, S. Peterson, K.-I. Sakakibara, H. Imagawa, 
A. Yamauchi, T. Nito, T. Yamasoba, N. Tayama, Estimation of inferiorsuperior vocal fold kinematics from high-speed stereo endoscopic data in vivo, J Acoust Soc Am 136 (2014) 3290-3300.

[7] G. Luegmair, D. Mehta, J. Kobler, Döllinger, Three-dimensional optical reconstruction of vocal fold kinematics using high-speed video with a laser projection system, IEEE Trans. Medical Imaging 34 (2015) 25722582 .

[8] M. Semmler, S. Kniesburges, V. Birk, A. Ziethe, P. Patel, M. Döllinger, 3D reconstruction of human laryngeal dynamics based on endoscopic high-speed recordings, IEEE Trans. Medical Imaging 35 (2016) 16151624 .

[9] A. Bouvet, A. Amelot, X. Pelorson, S. Maeda, A. Van Hirtum, External lighting and sensing photoglottography: characterization and MSePGG algorithm, Biomedical Signal Processing and Control 51 (2019) 318-327.

[10] A. Bouvet, I. Tokuda, X. Pelorson, A. Van Hirtum, Influence of level difference due to vocal folds angular asymmetry on auto-oscillating replicas, J. Acoust. Soc. 147 (2020) 1136-1145.

[11] B. Pickup, S. Thomson, Flow-induced vibratory response of idealized versus magnetic resonance imaging-based synthetic vocal fold models, J Acous Soc Am 128 (2010) 124-129.

[12] P. Murray, S. Thomson, Synthetic, multi-layer, self-oscillating vocal fold model fabrication, J Vis Exp 58 (2011) e3498.

[13] P. Murray, S. Thomson, Vibratory responses of synthetic, self-oscillating vocal fold models, J Acous Soc Am 132 (2012) 3428-3438.

[14] I. Tokuda, R. Shimamura, Effect of level difference between left and right vocal folds on phonation: Physical experiment and theoretical study, J Acous Soc Am 142 (2017) 482-492.

[15] R. Shimamura, I. T. Tokuda, Experimental study on level difference between left and right vocal folds, Acoustical Science and Technology 38 (2017) 264-267. 
[16] A. Bouvet, Experimental and theoretical contribution to the analysis and the modelling of the vocal folds vibration, Ph.D. thesis, Grenoble Alpes University, France, 2019.

[17] C. Pinho, L. Jesus, A. Barney, Aerodynamic measures of speech in unilateral vocal fold paralysis (UVFP) patients, Logopedics Phoniatrics Vocology 38 (2013) 19-34.

[18] A. Schindler, A. Bottero, P. Capaccio, D. Ginocchio, F. Adorni, F. Ottaviani, Vocal improvement after voice therapy in unilateral vocal fold paralysis, J Voice 22 (2008) 113-118.

[19] J. G. Svec, On vibration properties of human vocal folds, Ph.D. thesis, University of Groningen, 2000.

[20] R. Scherer, D. Shinwari, K. De Witt, C. Zhang, B. Kucinschi, A. Afjeh, Intraglottal pressure profiles for a symmetric and oblique glottis with a divergence angle of 10 degrees, J Acous Soc Am 109 (2001) 1616-1630.

[21] D. Mehta, M. Zanartu, T. Quatieri, D. Deliyski, R. Hillman, Investigating acoustic correlates of human vocal fold vibratory phase asymmetry through modeling and laryngeal high-speed videoendoscopy, J Acous Soc Am 105 (2011) 3999-4009.

[22] J. Jiang, Y. Zhang, M. Kelly, E. Bieging, M. Hoffman, An automatic method to quantify mucosal waves via videokymography, Laryngoscope 118 (2008) 1504-1510.

[23] M. J. Regner, M. Robitaille, J. Jiang, Interspecies comparison of mucosal wave properties using high-speed digital imaging, Laryngoscope 120 (2010) 1188-1194.

600 [24] S. Kumar, K. Phadke, J. Vydrova, A. Novozamsky, A. Zita, B. Zitova, J. Svec, Visual and automatic evaluation of vocal fold mucosal waves through sharpness of lateral peaks in high-speed videokymographic images, J voice 34 (2020) 170-178. 